\title{
ON MODELLING OF COMPLEX NETWORKS
}

\author{
Svetlana Atslega ${ }^{1,2}$, Felix Sadyrbaev ${ }^{2}$, Inna Samuilik ${ }^{3}$ \\ ${ }^{1}$ Latvia University of Life Sciences and Technologies, Latvia; \\ ${ }^{2}$ Institute of Mathematics and Computer Science, University of Latvia, Latvia; \\ ${ }^{3}$ Daugavpils University, Latvia \\ svetlana.atslega@1lu.lv, felix@latnet.lv,inna.samuilika@rtu.lv
}

\begin{abstract}
A network is considered, which is modelled by a system of ordinary differential equations. The dynamics of a network depends on the attracting sets in a phase space. The problem of control and management of this network is in a focus of our study. The specific case is considered, where attractors are periodic trajectories. We provide the way of controlling the network by changing of a certain group of parameters. The numerical approach combined with the analytical solutions is used. As a by-product, the existence of multiple periodic solutions is proved. They are constructed explicitly for a specific three-dimensional system. Problems of control and management of systems of this kind are challenging issues in the theory of genetic networks.
\end{abstract}

Keywords: nullclines, critical points, attracting sets.

\section{Introduction}

We would like to start with the citation "Scientists aim to understand, whereas engineers aim to modify and control" [1]. We do hope that in our short remark both "objectives come together in the context of genomic" regulatory networks (GRN in short). Nonlinear dynamical processes are often at the centre of the study in natural and engineering systems. It was pointed out [2] that in spite of significant progress in understanding and analysing large complex systems the problems of managing and control of such systems remain an outstanding challenge in interdisciplinary research. The principles of linear controllability [3] in general are not applicable to nonlinear dynamical networks. The linear theory defines that the network system is controllable if it is possible to drive the system from an arbitrary initial state to any final state in finite time. For nonlinear systems, control and management generally need to be specific and system dependent [2]. In this work the scheme of restricted control over the network, that have multiple stable attractors, was discussed. The network taken for the study is a realistic biological network, "T cells in large granular lymphocyte leukaemia associated with blood cancer". A network model considered in [2], contains 60 nodes and 195 regulatory edges. It was found that this network has three attractors, of which two correspond to two distinct cancerous states (denoted as $C 1$ and $C 2$ ) and one is associated with the normal state (denoted as $N$ ). Forty eight edges (connections) from the cancerous states to the normal one were discovered. The proper selection of the respective forty eight parameters can drive the system to the normal state. The existence of needed parameter perturbation was acknowledged. The attractor network was considered and the main proposition was to arrange experimental adjustment of parameters in order to achieve the required goal.

We consider below relatively simple model of three element GRN, which is described by the system or ordinary differential equations $[4 ; 5]$.

$$
\left\{\begin{array}{l}
\frac{d x_{1}}{d t}=\frac{1}{1+e^{-\mu_{1}\left(w_{11} x_{1}+w_{12} x_{2}+w_{12} x_{2}-\theta_{1}\right)}}-v_{1} x_{1}, \\
\frac{d x_{2}}{d t}=\frac{1}{1+e^{-\mu_{2}\left(w_{21} x_{1}+w_{22} x_{2}+w_{23} x_{3}-\theta_{2}\right)}}-v_{2} x_{2}, \\
\frac{d x_{3}}{d t}=\frac{1}{1+e^{-\mu_{3}\left(w_{31} x_{1}+w_{32} x_{2}+w_{33} x_{3}-\theta_{2}\right)}}-v_{3} x_{3},
\end{array}\right.
$$

where $\mu, \theta$ and $v$-parameters,

$w_{i j}$ - elements of the regulatory matrix.

$$
W=\left(\begin{array}{lll}
w_{11} & w_{12} & w_{13} \\
w_{21} & w_{22} & w_{23} \\
w_{31} & w_{32} & w_{33}
\end{array}\right) .
$$

The vector $\boldsymbol{x}(t)=\operatorname{col}\left(x_{1}(t), x_{2}(t), x_{3}(t)\right)$ describes the current state of a network. The trajectory $\left\{x(t): t \geq t_{0}, x\left(t_{0}\right)=x_{0}\right\}$ describes evolution of the state of a network, which satisfies the initial condition $x\left(t_{0}\right)=x_{0}$. The nonlinearity in (1) is represented by a sigmoidal function 


$$
f(z)=\frac{1}{1+e^{-\mu z}}
$$

This function monotonically increases from 0 to 1 as the argument $\mathrm{z}$ changes from $-\infty$ to $+\infty$. Systems of that type are used in the theory of neural networks $[4 ; 5]$, telecommunication networks [6] and genomic networks $[2 ; 6 ; 7]$. The main problem considering these type systems, is description of attractors, their dependence on parameters, control and management. The mathematical problems paralleling the above mentioned are: description and classification of possible attractors, clarification and roles of groups of parameters, behaviour of trajectories under the change of adjustable parameters and for different types of the regulation $W$. For practical purposes the structure of the phase space should be known in terms of various parameters.

One of our goals is to show how knowledge of the structure of the phase space can serve to manage and control the network. For this, we took our motivation from the work [2], where the authors had combined the efforts of biologists and mathematicians to clarify the situation. Namely, the reaction of the genomic network to progressing of lymphocyte leukaemia associated with blood cancer is considered and the ways of changing the situation are discussed. Generally, the disease state is identified with the attractive critical point of the system. The trajectories that are in a basin of attraction, naturally tend to this equilibrium. To redirect the trajectory to a normal state. Something should be done. This something can be expressed in terms of tuning the system using adjustable parameters. In our example we use theta parameters to control the system. Our analysis is mainly geometrical, using the zero isoclines (nullclines) of the system. We can move nullclines by changing $\theta$, thus eliminating the bad attractor for a period of time and waiting for the vector $\boldsymbol{x}(t)$ to enter the basin of attraction of needed attractor. This is our scheme and plan of action in short.

Another aspect of our treatment is usage of attractors that are not critical points. The specific feature of systems like (1) is that nullclines meet only in a bounded domain $G$, which is invariant with respect to the system (1). Therefore, all attractors are in $G$ and every critical point can be in G only. In our previous communication [8] we emphasized the role of the Andronov - Hopf bifurcation in emergency of periodic trajectories for 2-dimensional systems.

We show how three periodic trajectories can appear in three-dimensional (3D in short) system (1). We examine the behaviour of solutions and show that two of periodic solutions are stable limit cycles, which attract almost all the trajectories in $G$. We show what happens if the regulatory matrix changes its structure and the system (1) becomes coupled. Our study has connections with papers [9-12], where similar problems were studied. The novelty of the current paper is showing how periodic solutions can appear in 3D system and what are the conditions for their existence. The main feature is that we propose the method of controlling the system by changing $\theta$-parameters.

\section{Materials and methods}

Our consideration is mainly geometrical. All processes of interest to us take place in a bounded parallelepiped $\mathrm{G}$ and our main intent is to use the isocline method for understanding and managing the system. The nullclines of system (1) are given by the equations

$$
\left\{\begin{array}{l}
x_{1}=\frac{1}{v_{1}} \cdot \frac{1}{1+e^{-\mu_{1}\left(w_{11} x_{1}+w_{12} x_{2}+w_{12} x_{2}-\theta_{1}\right)}}, \\
x_{2}=\frac{1}{v_{2}} \cdot \frac{1}{1+e^{-\mu_{2}\left(w_{21} x_{1}+w_{22} x_{2}+w_{23} x_{3}-\theta_{2}\right)}}, \\
x_{3}=\frac{1}{3} \cdot \frac{1}{1+e^{-\mu_{3}\left(w_{31} x_{1}+w_{32} x_{2}+w_{33} x_{3}-\theta_{3}\right)}} .
\end{array}\right.
$$

It follows from (3) that the first nullcline is in the set

$$
\left\{\left(x_{1}, x_{2}, x_{3}\right): 0<x_{1}<\frac{1}{v_{1}},\left(x_{2}, x_{3}\right) \in R^{2}\right\}
$$

the second nullcline is in the set

$$
\left\{\left(x_{1}, x_{2}, x_{3}\right): 0<x_{2}<\frac{1}{v_{2}},\left(x_{1}, x_{3}\right) \in R^{2}\right\}
$$

and the third one is in the set 


$$
\left\{\left(x_{1}, x_{2}, x_{3}\right): 0<x_{3}<\frac{1}{v_{3}},\left(x_{1}, x_{2}\right) \in R^{2}\right\} .
$$

Therefore, all critical points are located in the open parallelepiped

$$
\left\{\left(x_{1}, x_{2}, x_{3}\right): 0<x_{1}<\frac{1}{v_{1}}, 0<x_{2}<\frac{1}{v_{2}}, 0<x_{3}<\frac{1}{v_{3}}\right\}=: G \text {. }
$$

There exists at least one critical point.

The type of a critical point can be detected in a standard way, using the linearized system and analyzing the characteristic equation.

\section{D periodic solutions}

Set the regulatory matrix to

$$
W=\left(\begin{array}{ccc}
w_{11} & w_{12} & 0 \\
w_{21} & w_{22} & 0 \\
0 & 0 & w_{33}
\end{array}\right)
$$

The system (1) is then uncoupled and consists of the $2 \mathrm{D}$ (two-dimensional) system

$$
\left\{\begin{array}{l}
\frac{d x_{1}}{d t}=\frac{1}{1+e^{-\mu_{1}\left(w_{11} x_{1}+w_{12} x_{2}-\theta_{1}\right)}}-v_{1} x_{1}, \\
\frac{d x_{2}}{d t}=\frac{1}{1+e^{-\mu_{2}\left(w_{21} x_{1}+w_{22} x_{2}-\theta_{2}\right)}}-v_{2} x_{2}
\end{array}\right.
$$

and a single equation

$$
\frac{d x_{3}}{d t}=\frac{1}{1+e^{-\mu_{3}\left(w_{33} x_{3}-\theta_{3}\right)}}-v_{3} x_{3}
$$

The third nullcline is defined by the equation

$$
x_{3}=\frac{1}{v_{3}} \cdot \frac{1}{1+e^{-\mu_{3}\left(w_{33} x_{3}-\theta_{3}\right)}},
$$

which may have up to three roots. If this is the case (we suppose it is), then the third nullcline is just a union of three parallel planes in the domain Q. Suppose that the system (5) has a stable limit cycle that have appeared as a result of Andronov - Hopf bifurcation like it was shown in [8].

For specific regulatory matrices of the form $w_{11}=w_{22}=k>0, w_{12}=2, w_{21}=-2$ sufficiently small nullclines intersect only once, and the respective critical point is stable focus. Under the increase of $k$ the single critical point changes to unstable focus and a periodic solution emerges. So, the periodic solutions that are used below in 3D examples are detected not numerically only, but exist in accordance with theoretical considerations. Then all three nullclines are located as shown in Figure 1.

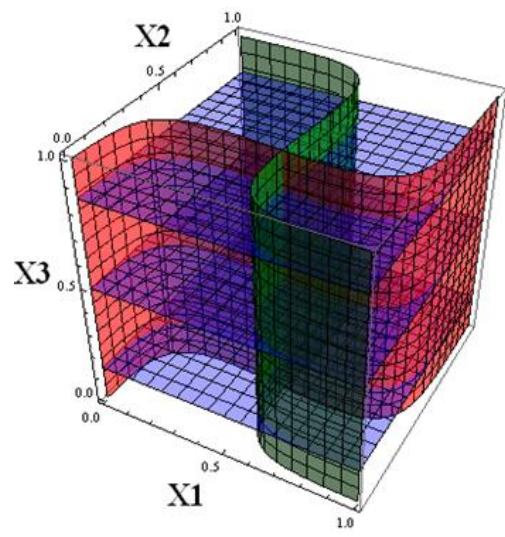

Fig. 1. $\mu_{1}=\mu_{2}=\mu_{3}=5, \theta_{1}=1.2, \theta_{2}=-0.6, \theta_{3}=0.5, W=\left(\begin{array}{ccc}1 & 2 & 0 \\ -2 & 1 & 0 \\ 0 & 0 & 1\end{array}\right)$ 
For these parameters the applicates of blue planes are $\mathrm{z}_{1}=0.1448$ (rounded), $\mathrm{z}_{2}=0.5$ (exact), $\mathrm{Z}_{3}=0.8552$ (rounded). So, there are exactly three critical points $p_{1}, p_{2}$ and $p_{3}$ at $(0.536688,0.346358$, $0.1448),(0.536688,0.346358,0.5),(0.536688,0.346358,0.8552)$, respectively.

Linearization around these points provides us with the characteristic numbers $\lambda$ given in Table 1 .

Table 1

\section{Characteristic numbers $\lambda$}

\begin{tabular}{|c|c|c|c|}
\hline- & $\lambda_{1}$ & $\lambda_{2}$ & $\lambda_{3}$ \\
\hline$p_{1}$ & -0.991643 & $0.18762-2.37198$ & $0.18762+2.37198$ \\
\hline$p_{2}$ & 2.75 & $0.18762-2.37198$ & $0.18762+2.37198$ \\
\hline$p_{3}$ & -0.991643 & $0.18762-2.37198$ & $0.18762+2.37198$ \\
\hline
\end{tabular}

There are three periodic solutions of the system (5), (6), lying in $G$ and in the planes $x_{3}=z_{1}, x_{3}=z_{2}$, $x_{3}=z_{3}$. This follows from our construction. Other solutions that are not in the middle plane $\left(z_{2}=0.5\right)$ tend to the upper or to the lower periodic solutions depicted in red. Solutions starting at the middle plane, tend to the middle periodic solution. It is generally unstable and is destroyed under small perturbation of the system (5), (6).

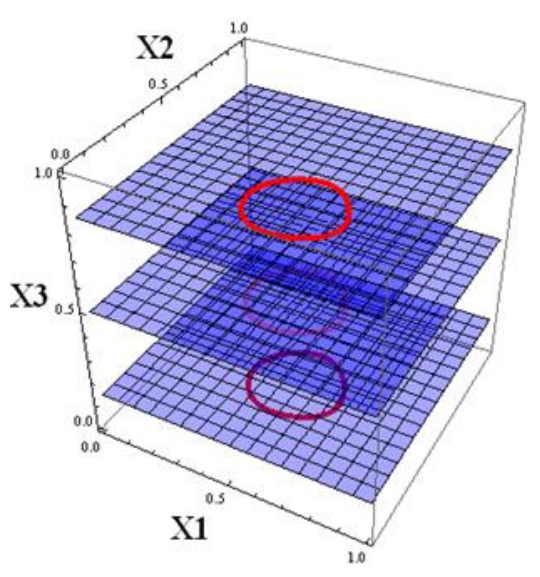

Fig. $2 . z_{1}=\mathbf{0 . 1 4 4 8}, z_{2}=\mathbf{0 . 5}, z_{3}=\mathbf{0 . 8 5 5 2}$

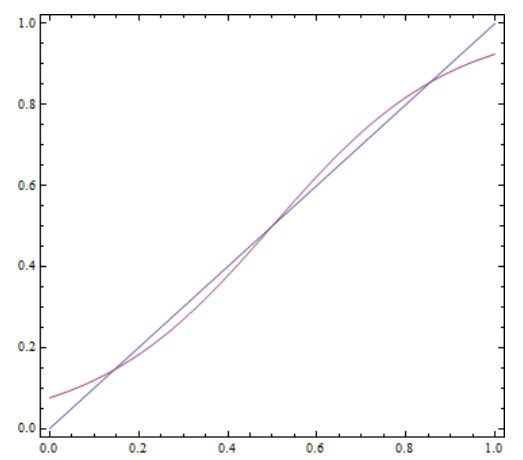

Fig. 4. $\theta_{3}=\mathbf{0 . 5}$

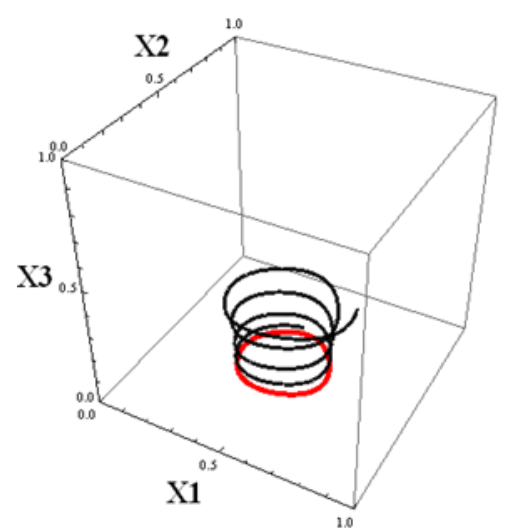

Fig. 3. Red - periodic solution for $z_{1}=\mathbf{0 . 1 4 4 8}$; black - trajectory of system (10), (11) with the initial conditions $(0.8,0.4,0.45)$

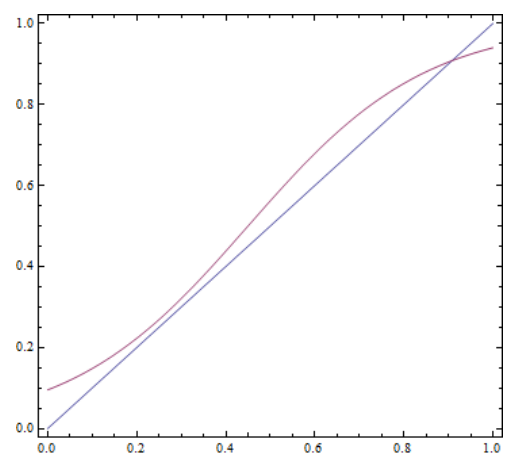

Fig. 5. $\theta_{3}=\mathbf{0 . 4 5}$

In Fig.4 and Fig. 3 respectively three and one solutions are detected for the equation (7) which defines z-nullclines, plots are

$$
\frac{1}{v_{3}} \cdot \frac{1}{1+e^{-\mu_{3}\left(w_{33} x_{3}-\theta_{3}\right)}}
$$

against $x_{3}$. 


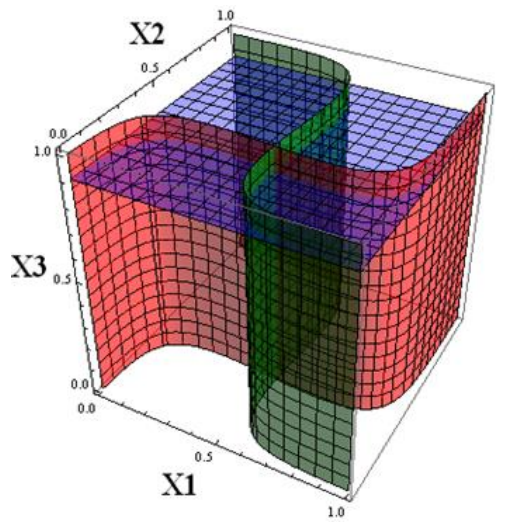

Fig. 6. $\mu_{1}=\mu_{2}=\mu_{3}=5, \theta_{1}=\mathbf{1 . 2}, \theta_{2}=\mathbf{- 0 . 6}$, $\theta_{3}=0.45, x_{3}=0.908$

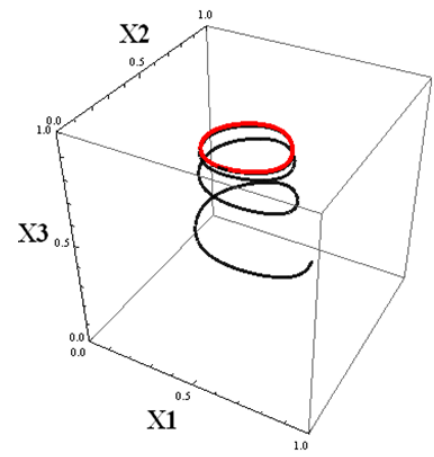

Fig. 7. Red - periodic solution for $x_{3}=\mathbf{0 . 9 0 8}$;

black - trajectory of system $(10),(11)$ with the initial conditions $(0.8,0.4,0.45)$

We have changed $\theta_{3}$ from 0.5 to 0.45 . Nullclines are now in Fig. 6 . The trajectory with the initial conditions $(0.8,0.4,0.45)$ goes to the periodic solution depicted in Fig. 7 . After some finite time it enters basin of attraction of the upper periodic solution, depicted in Fig. 2, and after restoring the value $\theta_{3}=0.5$, the state of the system tends to the "normal" attractor.

\section{Conclusions}

Genetic engineering will be an effective way of treatment diseases of living organisms. Knowledge of principles and in any practical case details of specific gene networks is needed for successful treatment and management of GRN. Mathematical models, if adequately describing GRN, can provide useful material for experiments and studying. We have shown how the three-dimensional model can be controlled by a simple change of one of the parameters. It is possible to generalize results to highdimensional systems and networks, considering systems with 2 n-dimensional regulatory matrices, containing low-dimensional blocks of the form (19).

For the mathematical model (1) we have shown that: 1) 3D system (1) can have several periodic trajectories (two stable ones and one semi-stable); 2) 3D system (1) can have no stable (attractive) critical points (like the famous Lorenz system); 3) system (1) can be managed by changing parameters $\left.\theta_{i} ; 4\right)$ the attractor for system (1) can contain two periodic trajectories; 5) any trajectory, going to one of the attractive periodic regimes, can be redirected to another one by simple changing of $\theta_{1}$ or $\theta_{3}$.

Generally, control and management for 3D systems is possible. Knowledge of critical points and basins of attraction of all attractors is needed for this.

\section{References}

[1] Abdallah C.T. Mathematical controllability of genomic networks. PNAS October 18, 2011, 108 (42) pp. 17243-17244; DOI: 10.1073/pnas.1114108108

[2] Wang, L.-Z., Su, R.-Q., Huang, Z.-G., Wang, X., Wang, W.-X., Grebogi, C., Lai, Y.-C. A geometrical approach to control and controllability of nonlinear dynamical networks. Nature Communications.Volume 7, 14 April 2016, Article number 11323.

[3] Kalman, R. E. Mathematical description of linear dynamical systems. J. Soc. Indus. Appl. Math. Ser. A 1, 1963, pp. 152-192.

[4] Wilson HR, Cowan JD. Excitatory and inhibitory interactions in localized populations of model neurons. Biophys J., vol 12 (1), 1972, pp. 1-24.

[5] Noonburg V.W. Differential Equations: From Calculus to Dynamical Systems, Providence, Rhode Island: MAA Press, 2019, $2^{\text {nd }}$ edition.

[6] Furusawa C., Kaneko K. A generic mechanism for adaptive growth rate regulation. PLoS Comput Biol. 2008; 4(1): e3. DOI: 10.1371/journal.pcbi.0040003.

[7] Jong H.D. Modeling and Simulation of Genetic Regulatory Systems: A Literature Review. J. Computational Biology, vol 9 (1), 2002, pp. 67-103. 
[8] Atslega, S., Sadyrbaev, F. On modelling of artificial networks arising in applications. Engineering for Rural Development, 2020, 19, pp. 1659-1665

[9] Atslega S., Finaskins D., Sadyrbaev F. On a Planar Dynamical System Arising in the Network Control Theory. Math. Modelling and Analysis, vol. 21 (3), 2016, pp. 385-398.

[10] Brokan E., Sadyrbaev F. On a differential system arising in the network control theory. Nonlinear Analysis: Modelling and Control, vol. 21 (5), 2016, pp. 687-701.

[11] Brokan E., Sadyrbaev F. Attraction in $n$-dimensional differential systems from network regulation theory. Mathematical Methods in Applied Sciences, vol. 41 (17), 2018, pp. 7498-7509.

[12] Ogorelova D, Sadyrbaev F, Sengileyev V. Control in Inhibitory Genetic Regulatory Network Models. Contemporary Mathematics [Internet]. 2020Oct.22 [cited 2021Jan.26];1(5). 